\title{
4. Diagnóstico diferencial de la fibrosis pulmonar idiopática
}

\author{
GEORGINA MIRANDA S.* y MAURICIO SALINAS F.**
}

\section{Differential diagnosis of idiopathic pulmonary fibrosis}

For the accurate diagnosis of idiopathic pulmonary fibrosis (IPF), the presence of a definitive tomographic pattern of usual interstitial pneumonia (UIP) is of vital importance, in an appropriate clinical context. Targeted interrogation, the use of valid questionnaires, an acute rheumatologic evaluation and complementary examinations are important to rule out secondary causes such as hypersensitivity pneumonitis (HP), connective tissue diseases (CTD), drug toxicity and some pneumoconiosis that can mimic the radiological pattern and often hinder a clear diagnosis of IPF.

Key words: Idiopathic pulmonary fibrosis; Alveolitis, Extrinsec Allergic; Pneumoconiosis; Connective Tissue Diseases; Drug-Related Side Effects and Adverse Reactions; Surveys and Questionnaires.

\section{Resumen}

Para el diagnóstico certero de fibrosis pulmonar idiopática (FPI) es de vital importancia la presencia de un patrón tomográfico definitivo de neumonía intersticial usual (NIU), en un contexto clínico adecuado. El interrogatorio dirigido, el uso de cuestionarios validados, una evaluación reumatológica acuciosa y exámenes complementarios son importantes para descartar causas secundarias de fibrosis pulmonar como neumonitis por hipersensibilidad (NHS), enfermedades del tejido conectivo (ETC), toxicidad por drogas y algunas neumoconiosis que pueden imitar el patrón radiológico y muchas veces dificultar un diagnóstico adecuado de FPI.

Palabras clave: Fibrosis Pulmonar Idiopática; Alveolitis Alérgica Extrínseca; Neumoconiosis; Enfermedades del Tejido Conectivo; Efectos Colaterales y Reacciones relacionadas a Drogas; Encuestas y Cuestionarios.

Frente a la sospecha de fibrosis pulmonar idiopática (FPI), los siguientes criterios deben aplicarse para el diagnóstico ${ }^{1}$ :

1. Exclusión de otras causas de enfermedad pulmonar intersticial (EPI), exposiciones ambientales ocupacionales y domésticas, enfermedades del tejido conectivo, toxicidad por drogas.

2. La presencia de patrón tomográfico definitivo de neumonía intersticial usual (NIU).

3. Combinaciones específicas de patrones tomográficos e histopatológicos en pacientes sometidos a biopsia pulmonar.
Es necesaria la presencia del punto 1 más punto 2 o 3 para el diagnóstico definitivo de NIU.

En muchas oportunidades este algoritmo no es tan sencillo y es necesaria la evaluación de un equipo multidisciplinario para el diagnóstico definitivo.

El protocolo de tomografía computada de alta resolución (TCAR) de tórax implica, idealmente, un estudio volumétrico de alta resolución, en supino en fin de inspiración, en supino en fin de espiración y, en algunos casos, en prono en fin de inspiración (el análisis de las imágenes en FPI se describe en detalle en el capítulo 5).

* Hospital Padre Hurtado, Clínica Alemana de Santiago y Universidad del Desarrollo. Santiago, Chile.

** Instituto Nacional del Tórax, Facultad de Medicina Universidad de Chile. Clínica Santa María. Santiago, Chile. 
El diagnóstico diferencial de FPI más frecuente y difícil es el de neumonitis por hipersensibilidad crónica (NHS) crónica ${ }^{2}$. Es por esto que es importante obtener una historia estandarizada con énfasis en exposiciones ambientales permanentes, en casa, trabajo o pasatiempos a elementos fibrogénicos como hongos, aves, plumones, animales, polvo de metales (latón, plomo, acero), polvo de madera (pino), polvo vegetal, exposición a ganado, corte y pulido de piedra. Conviene hacer uso de cuestionarios validados, idealmente adaptados a la realidad de cada región. En el Anexo 2, se adjunta cuestionario modificado de NHS de la Universidad de California en San Francisco (UCSF) ${ }^{3}$.

Si bien el antecedente de exposición es de vital importancia en la sospecha de NSH crónica, en hasta un $60 \%$ de los casos no es posible identificarla. Los estudios complementarios para identificar el antígeno (IgG específicas o precipitinas séricas) podrían tener un papel. Sin embargo, dados los problemas de sensibilidad y estandarización de estas técnicas, es controversial solicitarlas a todos los pacientes ${ }^{4}$.

En la historia clínica el antecedente de uso de fármacos es de mucha importancia. El posible papel de medicamentos neumotóxicos (p.j. amiodarona, nitrofurantoína, bleomicina, etc.) se puede revisar en la información contenida en la página www.pneumotox.com. Es fundamental buscar factores de riesgo FPI, como tabaquismo, historia familiar de fibrosis pulmonar (síndrome de telómeros cortos con anemia macrocítica, daño hepático crónico, canicie precoz) y enfermedad por reflujo gastroesofágico.

La fibrobroncoscopia (FBC) para lavado broncoalveolar (LBA) y/o realización de biopsia transbronquial es útil cuando existe la sospecha de un diagnóstico alternativo a FPI, en la evaluación de ciertas patologías más centradas en las cercanías de la vía aérea como neumonitis por hipersensibilidad o sarcoidosis. Sin embargo, su papel en FPI es limitado, por lo que no se sugiere su realización de forma rutinaria ${ }^{5,6}$.
La evaluación reumatológica de los pacientes en estudio por FPI se describe más adelante (capítulo 7). No existe consenso universal sobre qué serologías se deben solicitar y si se debe hacer tamizado a todos los pacientes o solo de acuerdo a la sospecha clínica-radiológica, lo cual depende también de los recursos disponibles. En nuestra opinión se debe ser exhaustivo en buscar los elementos clínicos de enfermedad reumatológica y conocer los patrones tomográficos propios de cada una de esas enfermedades. Frente a los casos dudosos o con elementos incompletos, el comité multidisciplinario debe ser el punto de discusión y acuerdo entre los distintos especialistas involucrados, para definir la mejor conducta o tratamiento para cada paciente.

\section{Bibliografía}

1.- RAGHU G, REMY-JARDIN M, MYERS JL, RICHELDI L, RYERSON CJ, LEDERER DJ, et al. Diagnosis of idiopathic pulmonary fibrosis: an official ATS/ERS/JRS/ALAT clinical practice guideline. Am J Respir Crit Care Med. 2018;198:e44-e68.

2.- VASAKOVA M, MORELL F, WALSH S, LESLIE K, RAGHU G. Hypersensitivity pneumonitis: perspectives in diagnosis and management. Am J Respir Crit Care Med 2017; 196: 680-9.

3.- https://www.ucsfhealth.org/pdf/initial_questionnaire_form_ild.pdf.

4.- FENOGLIO CM, REBOUX G, SUDRE B, MERCIER M, ROUSSEL S, CORDIER JF, et al. Diagnostic value of serum precipitins to mould antigens in active hypersensitivity pneumonitis. Eur Respir J 2007; 29: 706-12.

5.- HUNNINGHAKE GW, ZIMMERMAN MB, SCHWARTZ DA, KING TE JR, LYNCH J, HEGELE R, et al. Utility of a lung biopsy for the diagnosis of idiopathic pulmonary fibrosis. Am J Respir Crit Care Med 2001; 164: 193-6.

6.- BERBESCU EA, KATZENSTEIN AL, SNOW JL, ZISMAN DA. Transbronchial biopsy in usual interstitial pneumonia. Chest 2006; 129: 1126-31.

Correspondencia a:

Dra. Georgina Miranda S.

Email: gmirandasaez@gmail.com 